\title{
Spatial dynamic patterns of hand-foot-mouth disease in the People's Republic of China
}

\author{
Jin-Feng Wang ${ }^{1}$, Cheng-Dong Xu1 ${ }^{1}$, Shi-Lu Tong ${ }^{2}$, Hong-Yan Chen ${ }^{3}$, Wei-Zhong Yang ${ }^{4}$ \\ ${ }^{1}$ State Key Laboratory of Resources and Environmental Information Systems, Institute of Geographic Sciences \\ and Natural Resources Research, Chinese Academy of Sciences, Beijing, People's Republic of China; ${ }^{2}$ School of \\ Public Health and Institute of Health and Biomedical Innovation, Queensland University of Technology, \\ Brisbane, Australia; ${ }^{3}$ School of Environmental Sciences, University of Liverpool, Liverpool, United Kingdom; \\ ${ }^{4}$ Office for Disease Control and Emergency Response, Chinese Center for Disease Control and Prevention, \\ Beijing, People's Republic of China
}

\begin{abstract}
Hand-foot-mouth disease (HFMD) is the most common and widespread infectious disease in the People's Republic of China. Although there has been a substantial increase of HFMD in many parts of the country in recent years, its spatial dynamics and determinants remain unclear. When we collected and analysed weekly data on HFMD cases from 1,456 counties and the corresponding meteorological factors from 1 May 2008 to 27 March 2009, it was found that HFMD was spatially dispersed across the country in the summer and winter, while clustered in the spring and autumn. The spatial variation of HFMD was found to be affected by a combination of climate variables, while its spatio-temporal transmission was largely driven by temperature variations with a 7-week lag implying that (i) the dispersal of the disease can be anticipated based on the variation of the temperature and other climate variables; and (ii) the spatial dynamics of HFMD can be robustly predicted 7 weeks ahead of time using temperature data only. The findings reported allow prompt preparation and implementation of appropriate public health interventions to control and prevent disease outbreaks.
\end{abstract}

Keywords: hand-foot-mouth disease, spatial dynamic, spatio-temporal transmission, People's Republic of China.

\section{Introduction}

Hand-foot-mouth disease (HFMD) is a human syndrome including fever, blister-like sores in the mouth and a skin rash caused by intestinal viruses of the picornaviridae family, most commonly coxsackie A virus or enterovirus 71 (EV-71) (http://www. cdc.gov/hand-foot-mouth/). With 0.5-1 million reported cases annually, HFMD is the most frequent and wide spread infectious disease in the People's Republic of China (P.R. China). A number of epidemiological studies have been conducted to investigate its transmission parameters and identify pathogens across dif-

Corresponding authors:

Jin-Feng Wang

LREIS, Institute of Geographic Sciences and

Natural Resources Research, Chinese Academy of Sciences

Beijing, 100101, People's Republic of China

Tel. +86106488 8965 Fax +861064889630

E-mail: wangjf@igsnrr.ac.cn

Wei-Zhong Yang

Office for Disease Control and Emergency Response

Chinese Center for Disease Control and Prevention

Beijing 100050, People's Republic of China

Tel. +861083152988 Fax +861058900575

E-mail: yanwz@chinacdc.cn ferent time periods and in different locations (Hii et al., 2011; Wang et al., 2011a; Zhang et al., 2011). Previous studies have shown that the disease is a moderately transmittable infectious disease with an effective reproduction (the average number of secondary cases caused by each case, during the infectious period) of approximately 1.4, mainly among preschoolaged children. A higher risk of transmission is associated with temperatures in the range of $21^{\circ} \mathrm{C}$ to $27^{\circ} \mathrm{C}$, high relative humidity, low wind speed, high precipitation, high population density and periods during which schools are open (Wang et al., 2011a). Spatiotemporal HFMD clusters, closely linked to the monthly precipitation, have been found in P.R. China (Wang et al., 2011a). In Singapore, there is normally a 1-2 week delay in the incidence of HFMD in response to weekly temperature changes and rainfall (Hii et al., 2011). However, the spatial pattern of the disease is not yet fully understood.

The spatio-temporal features of an infectious disease is usually driven by determinants, which can provide invaluable information for exploring the risk factors of the disease and contribute to developing effective measures to control and prevent its transmission (Christakos, 2005; Wang et al., 2011a). Spatio-temporal analysis is increasingly used in epidemiological 
research based on specific or routinely collected data from different sources (Kulldorff, 1997; Haining, 2003; Riley, 2008). In a previous study, we used the "self organization mapping-Bayesian maximum entropy" (S-BME) technique (Christakos and Hristopulos, 1998; Kolovos et al., 2010) to explore the relationship between HFMD transmission and climate types on a moderate spatial scale (Wang et al., 2011). However, this approach appeared to overly compress the spatio-temporal information, sometimes masking spatio-temporal trends resulting in loss of critical information regarding the transmission process (Wang et al., 2011a). We applied the mass centre method (Wang, 2009) and Moran's I (Moran, 1950) to datasets of HFMD incidence and climatic drivers to describe their spatial distribution and spatial clustering at each "time slice" in a series of temporal data to illustrate the spatial dynamics of disease transmission. The purpose was to explore the spatio-temporal association between disease transmission and climate variation and to discuss policy implications with respect to intervention.

\section{Materials and methods}

We focused on 1,456 counties in the East because the western part of P.R. China is sparsely inhabited (Fig. 1). These counties comprise $90 \%$ of the nation's population. Between 1 May 2008 and 27 March 2009 , the average incidence of HFMD was 834.1 per 100,000 in these areas (Chinese Center for Disease Control and Prevention, Beijing). We collected the weekly average numbers of HFMD cases in the 1,456 counties as well as the weekly averages of the meteorological variables (the mean, maximum and minimum temperatures, air pressure, humidity, wind speed, precipitation and hours of sunshine). The disease data were reported by the county hospitals to the Chinese Center for Disease Control and Prevention, while the climate information was provided by the Chinese Meteorological Data Sharing Service System. The weekly averages of the meteorological variables were calculated based on the daily values recorded at 676 weather stations across the 1,456 counties.

Raw data usually contain too much noise to display

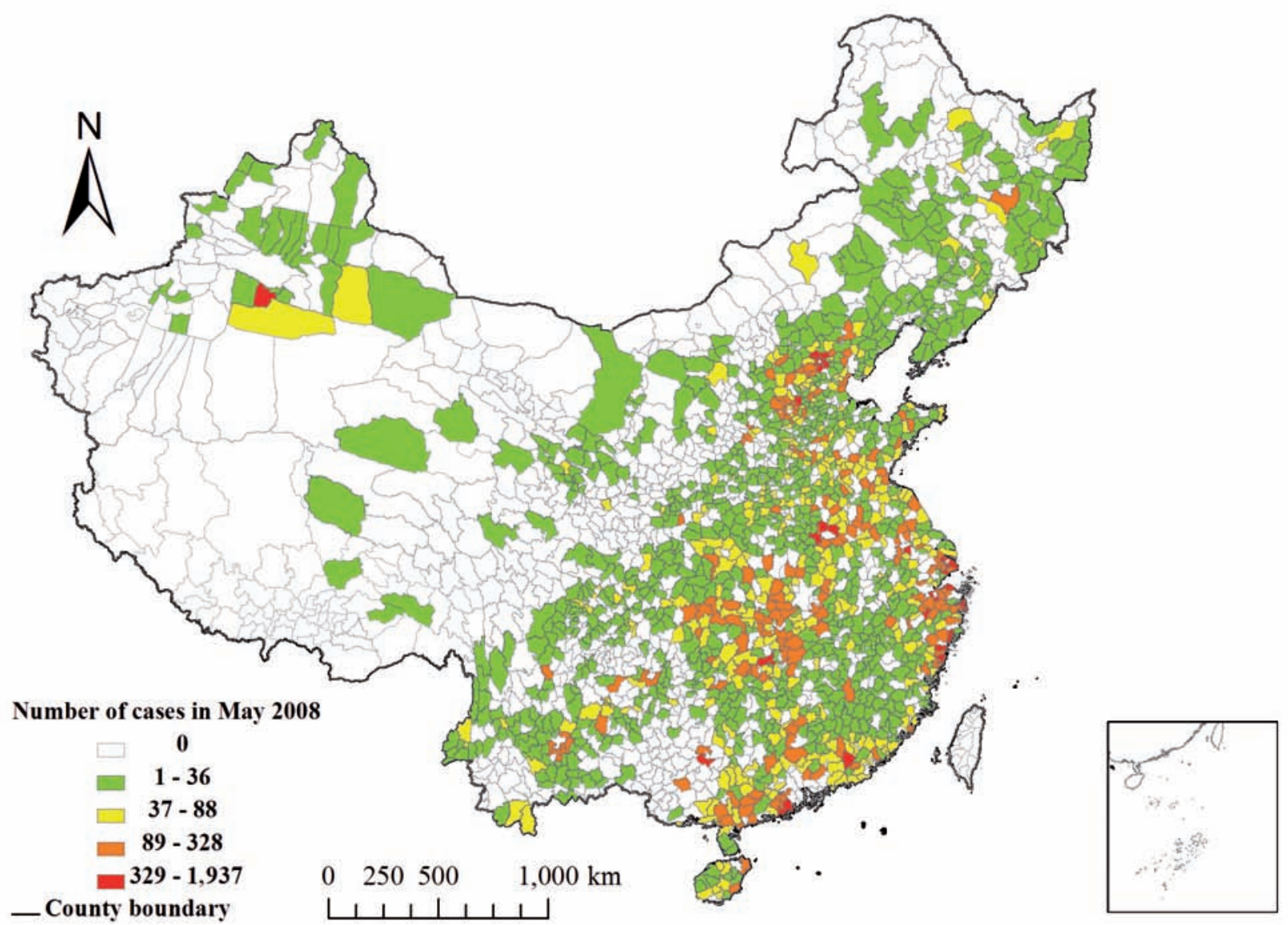

Fig. 1. Distribution of HFMD cases in P.R. China in May 2008. 
a pattern of a dynamic phenomenon. Conventional statistics, time series or spatial statistics are therefore often used to describe point estimates with either a temporal or a spatial domain deriving the features of the phenomenon in question from the analysis. Spatiotemporal statistics is becoming a mainstream approach to investigate the transmission and dispersal of infectious diseases (Riley, 2008; Zhou, 2009; Christakos, 2010; Wang et al., 2011a; Yu et al., 2011). However, because spatio-temporal processes are integrated and based on data from all domains, the study of a single domain would neglect information from the other domains. In the current study, spatial dynamics of both case and cluster distributions were examined by a series of spatial mass centres of and Moran's I statistic values. The association between HFMD with a single factor (first row in Fig. 2) and their interaction (second row in Fig. 2) were tested by the Fréchet distance approach (Alt and Godau, 1995) and the geographical detector method (Wang et al., 2010b, Wang and $\mathrm{Hu}, 2012$ ) (Fig. 2) comparing the time series of spatial clustering of HFMD and climate variables (third row in Fig. 2).

The Fréchet distance is the measure of similarity between two curves. This method takes into account the location and ordering of the points along the curves. A popular illustration of the Fréchet distance of two curves is the following: suppose a man (symbolizing the driving force of a disease) walking (spatial dynamics) his dog (the disease) on a leash (the association between the disease and its driving force); the man (the force) is walking (moving forward) along one curve (time series of the driver) and the dog (spatial dynamics of the disease) on another curve (time series of the disease). Seen in this way, the Fréchet distance is the shortest length of the leash (association) allowing them (the determinant and disease) to be on two separate curves from start to finish. Given two curves, $f:\left[a, a^{\prime}\right] \rightarrow V, g:\left[b, b^{\prime}\right] \rightarrow V$, $\delta_{F}(f, g)$, where $V$ stands for a Euclidean vector space, their Fréchet distance is defined as:

$$
\delta_{F}(f, g)=\inf _{\substack{\alpha[0,1] \rightarrow\left[a, a^{\prime}\right] \\ \beta[0,1] \rightarrow\left[b, b^{\prime}\right]}} \max _{\substack{t \in[0,1] \\ \text { and }}}\|f(\alpha(t))-g(\beta(t))\|
$$

where $\alpha(0)=a, \alpha(1)=a^{\prime}, \beta(0)=b$ and $\beta(1)=b$. The factor $\delta_{F}$ is obviously symmetric and the triangle inequality holds; the two curves are equivalent if the distance between them is zero (Alt and Godau, 1995). Two curves with different lengths $\left[a, a^{\prime}\right]$ and $\left[b, b^{\prime}\right]$ are standardised into the same length $[0,1]$, by two transfer functions $\alpha$ and $\beta$, with a parameter $t \in[0,1]$, the values of the two curves $f$ and $g$ are then coordinated by $\alpha(t)$ and $\beta(t)$ respectively, and their difference measured by $\delta_{F}(f, g)$ as defined by the equation above. The discrete Fréchet distance provides a good approximation of the continuous measure and can be efficiently computed using a simple algorithm (Eiter and Mannila, 1994). The similarity between two curves is determined by the equation:

$$
\begin{aligned}
& \inf _{\alpha[0,1] \rightarrow\left[a, a^{\prime}\right]} \max _{t \in[0,1]}\|f(\alpha(t))-g(\beta(t))\| \\
& S I(f, g)=1-\frac{\beta[0,1] \rightarrow\left[b, b^{\prime}\right]}{\sup _{\substack{\alpha[0,1] \rightarrow\left[a, a^{\prime}\right] \\
\beta[0,1] \rightarrow\left[b, b^{\prime}\right]}} \max _{\substack{t \in[0,1] \\
\text { and }}}\|f(\alpha(t))-g(\beta(t))\|}
\end{aligned}
$$

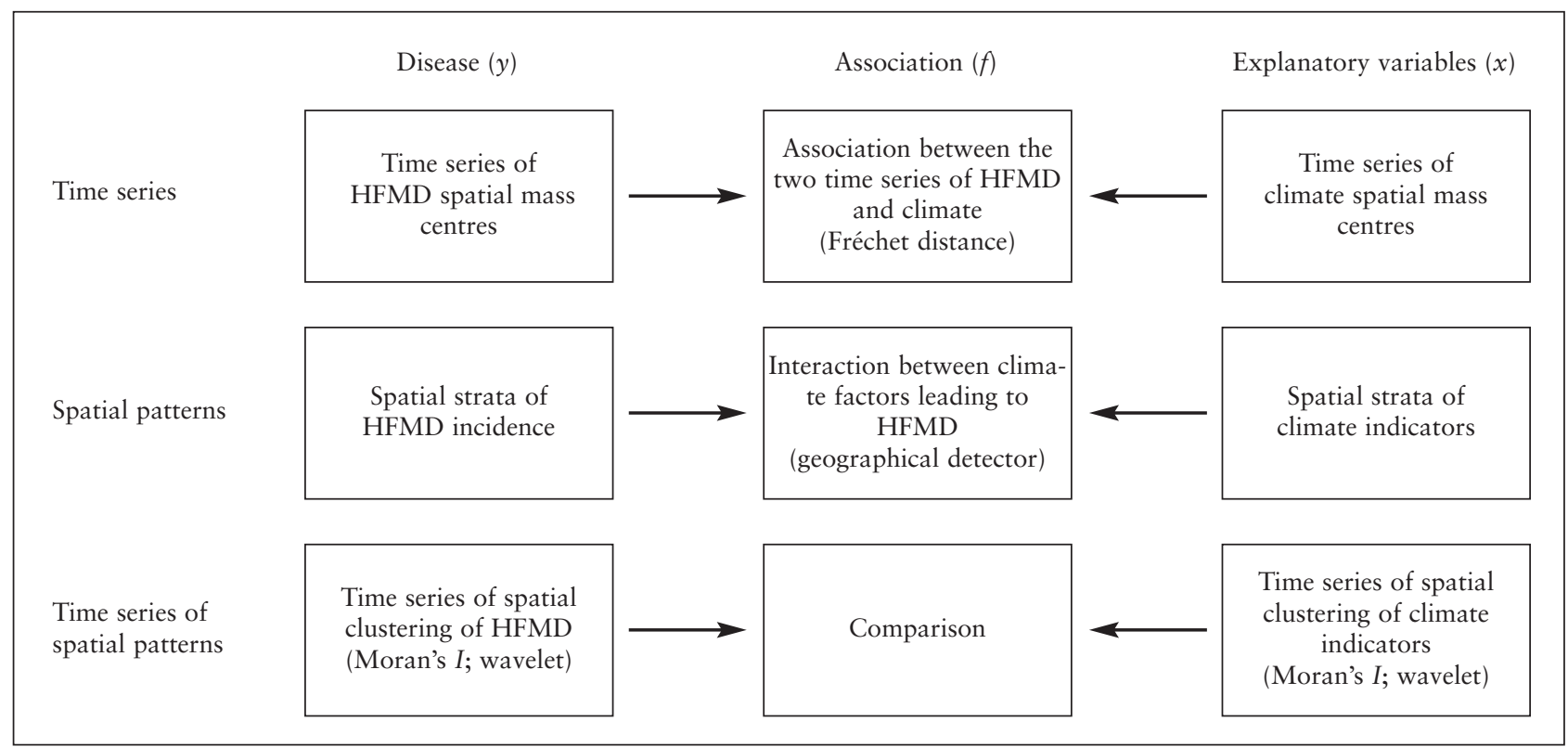

Fig. 2. Flow-chart of the study. 
where $S I(f, g) \in[0,1]$. A value near 1 indicates more similarity between the two curves, while a value near 0 indicates less similarity between them. $I$ standardizes $\delta_{F}$ into [0.1] in order to facilitate determining the degree of similarity between two curves in the limited bound.

The degree of global spatial data clustering was measured using Moran's I coefficient (Moran, 1950). The value of a Moran's $I$ lies between -1 and 1 , where the value near +1.0 indicates clustering of HFMD incidence or climate indictors, while -1.0 indicates spatial dispersal. Spatial clustering at time $t$ was obtained by:

$$
I(t)=\frac{N \sum_{i=1}^{N} \sum_{j=1}^{N} w_{i j}\left[y_{i}(t)-\bar{y}(t)\right]\left[y_{i}(t)\right]-\bar{y}(t)}{\left(\sum_{i=1}^{N} \sum_{j=1}^{N} w_{i j}\right) \sum_{j=1}^{N}\left[y_{i}(t)-\bar{y}(t)\right]^{2}}
$$

where $y_{i}(t)$ is HFMD incidence or climate indictors observed at location $i$ in week $t, \bar{y}$ the mean of a quantity such as the number of cases or a climate indicator averaged over the country in the $t$-th week, and $w_{i j}$ is the weight between two locations calculated by the inverse distance square method. The time series of $I(\mathrm{t})$ was often noisy, so it was filtered using a discrete wavelet transformation implemented by the Matlab computer library with Daubechies $\mathrm{db} 3$ as the mother-wave and four decomposition levels (http://www.mathworks.com). The time series was thus decomposed into low-frequency components $\left(a_{1}, a_{2}, a_{3}, a_{4}\right)$, reflecting their fundamental trend, and high frequency components $\left(d_{1}, d_{2}, d_{3}, d_{4}\right)$ reflecting the noise caused by random factors. The wavelet principle is explained by Fig. 5, where Moran's $I$ of HFMD is decomposed into $a_{1}$ and $d_{1}$; the trend of $a_{1}$ first not being clarified so it is further decomposed into $a_{2}$ and $d_{2}$ and so on until we reach $a_{4}$, in which the low frequency component $\left(a_{4}\right)$ clearly displays the spatial dispersion from August until late November. The wavelet decomposition of climate indicators in Fig. 5 can be interpreted in a similar way. The red lines mark the lowest spatial clustering of HFMD (in late November) and of the major climate indicators (in October), respectively.

A disease will exhibit a spatial distribution similar to that of an environmental factor, if the environmental attribute leads to the disease. This maxim has generated the geographical detector (Wang et al., 2010b) that can be applied for incidence prediction, since people living in different climate strata may have distinctly different incidences of HFMD. The geographical detector is grounded on the power of determinant
$(P D)$, which generates four detectors (Wang et al., $2010 \mathrm{~b})$ as follows from the equation:

$$
P D=1-\frac{1}{\Re \sigma^{2}} \sum_{b=1}^{L} \Re_{b} \sigma_{b}^{2}
$$

where $\Re$ and $\sigma^{2}$ denote the area and the variance of disease incidence of the study area, respectively. The study area is stratified into $L$ strata, denoted by $h=$ $1, \ldots, L$ (Wang et al., 2010a), according to spatial heterogeneity (defined as an attribute of which statistical properties, e.g. mean and standard deviation or change in space) of a suspected determinant or its proxy of the disease. $P D \in[0,1]$ means that $P D$ can vary between 1 (the determinant completely controls the disease) and 0 (the determinant is completely unrelated to the disease). Therefore, the $P D$ reflects the degree to which a determinant explains the disease prevalence. The geographical detector examines whether two health determinants, $A$ and $B$, together weaken or enhance one another, or whether they are independent in contributing to the development of a disease. The interaction between these two determinants can be justified by comparing the sum of disease contribution of two individual attributes with the contribution of the two attributes when combined, i.e.

$\begin{array}{lll}\text { They enhance } & \text { if } & P D(A \cap B)>P D(A) \text { or } P D(B) \\ \text { They enhance (bivariate) } & \text { if } & P D(A \cap B)>P D(A) \text { and } P D(B) \\ \text { They enhance (nonlinear) } & \text { if } & P D(A \cap B)>P D(A)+P D(B) \\ \text { They weaken } & \text { if } & P D(A \cap B)<P D(A)+P D(B) \\ \text { They weaken (univariate) } & \text { if } & P D(A \cap B)<P D(A) \text { or } P D(B) \\ \text { They weaken (nonlinear) } & \text { if } & P D(A \cap B)<P D(A) \text { and } P D(B) \\ \text { They are independent } & \text { if } & P D(A \cap B)=P D(A)+P D(B)\end{array}$

where the interaction (symbolised by $\cap$ ) can easily be implemented in a geographical information system (GIS) by overlaying the two factor layers $A$ and $B$. The geographical detector approach was implemented by using a computer package available at http://www.sssampling.org/geogdetector (Wang and $\mathrm{Hu}, 2012)$.

\section{Results}

Fig. 3 shows the mass centre tracks of HFMD incidence and the different climate indicators during the 47 weeks from 1 May 2008 to 26 March 2009, in which the mass centre disease tracks appear to follow the temperature variations. The relation between calendar date and the week number marked in Fig. 3 is shown in Table 1. 

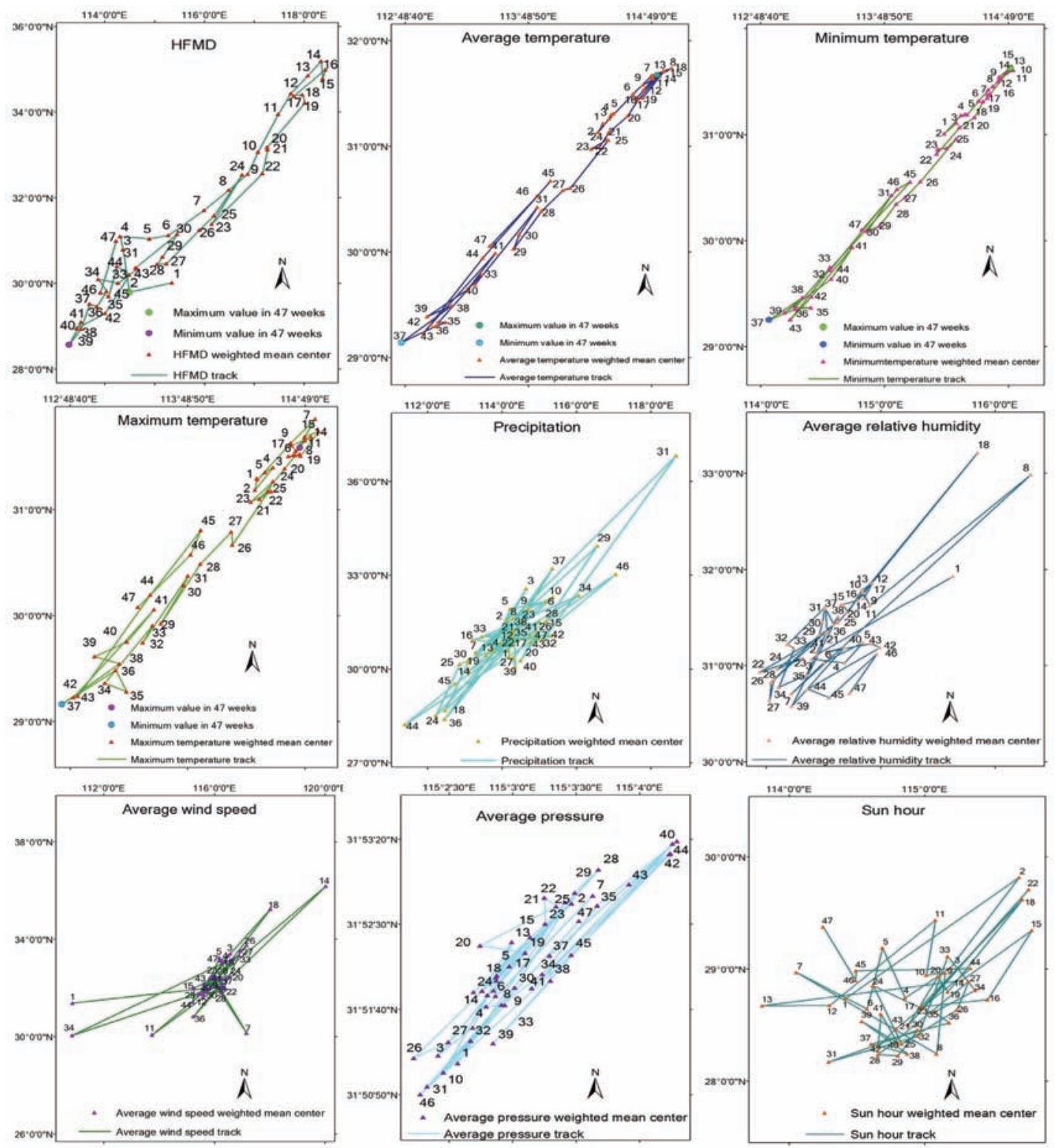

Fig. 3. HFMD mass centre movements and the climate indicators during the 47 -week period (numbers correpond to the weeks of Table 1).

Table 1. Correspondence between week number of the study period and calender date.

\begin{tabular}{lllllllllll}
\hline Week & 1 & 2 & 3 & 4 & 5 & 6 & 7 & 8 & 9 & 10 \\
Date* & $2008-5-1$ & $2008-5-8$ & $2008-5-15$ & $2008-5-22$ & $2008-5-29$ & $2008-6-5$ & $2008-6-12$ & $2008-6-19$ & $2008-6-26$ & $2008-7-3$ \\
Week & 11 & 12 & 13 & 14 & 15 & 16 & 17 & 18 & 19 & 20 \\
Date* & $2008-7-10$ & $2008-7-17$ & $2008-7-24$ & $2008-7-31$ & $2008-8-7$ & $2008-8-14$ & $2008-8-21$ & $2008-8-28$ & $2008-9-4$ & $2008-9-11$ \\
Week & 21 & 22 & 23 & 24 & 25 & 26 & 27 & 28 & 29 & 30 \\
Date* & $2008-9-18$ & $2008-9-25$ & $2008-10-2$ & $2008-10-9$ & $2008-10-16$ & $2008-10-23$ & $2008-10-30$ & $2008-11-6$ & $2008-11-13$ & $2008-11-20$ \\
Week & 31 & 32 & 33 & 34 & 35 & 36 & 37 & 38 & 39 & 40 \\
Date* & $2008-11-27$ & $2008-12-4$ & $2008-12-11$ & $2008-12-18$ & $2008-12-25$ & $2009-1-1$ & $2009-1-8$ & $2009-1-15$ & $2009-1-22$ & $2009-1-29$ \\
Week & 41 & 42 & 43 & 44 & 45 & 46 & 47 & & & \\
Date* & $2009-2-5$ & $2009-2-12$ & $2009-2-19$ & $2009-2-26$ & $2009-3-5$ & $2009-3-12$ & $2009-3-19$ & & &
\end{tabular}

"yyyy-mm-dd 


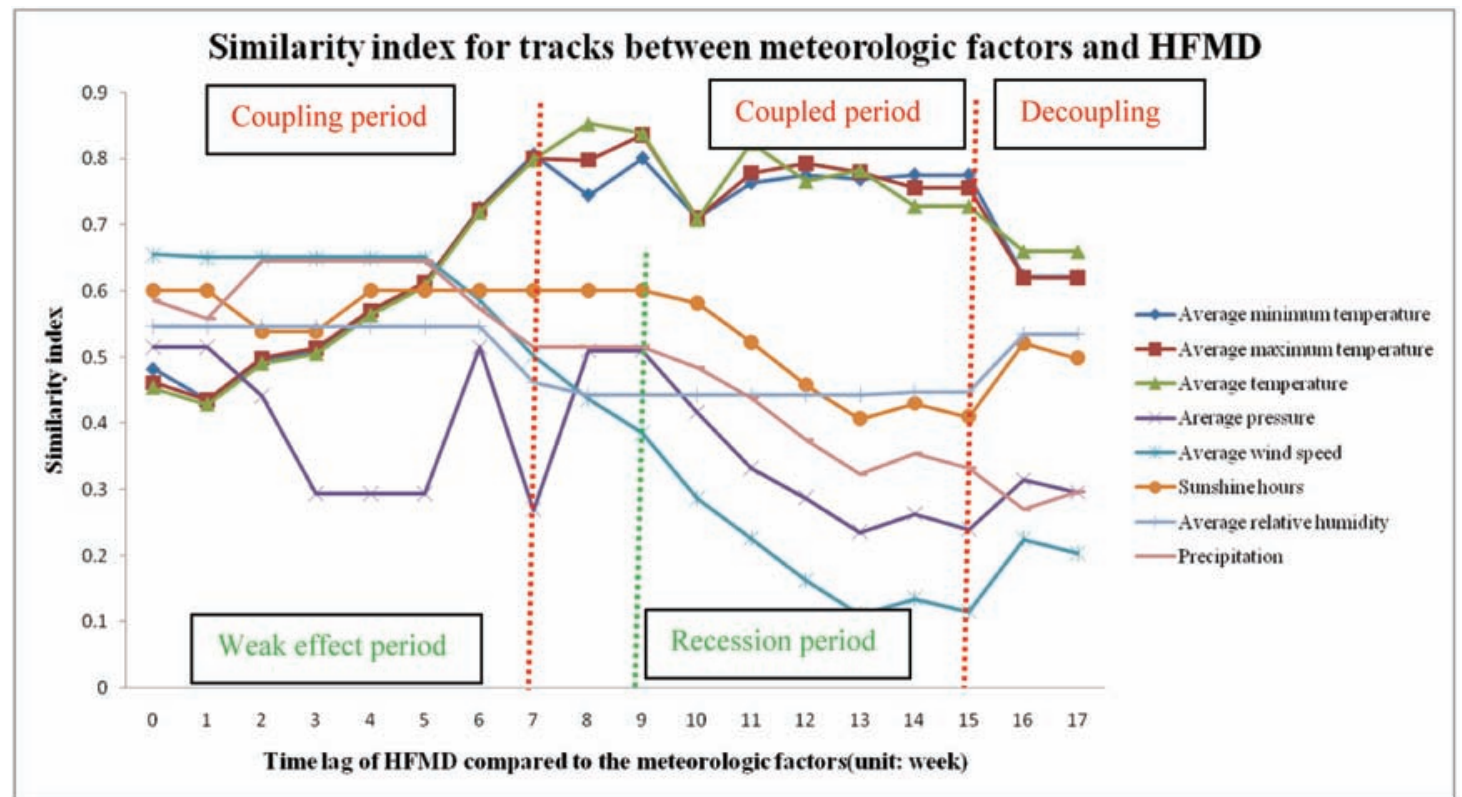

Fig. 4. Similarity indices between HFMD incidence and the various climate indicators. The X-axis denotes the time lag in weeks.

Fig. 4 shows that the incidence of HFMD and the variation pattern of temperature indictors (the mean, maximum and minimum temperatures) were well matched most of the time, although this similarity varied depending on the time lag. The similarity between HFMD and temperature increased significantly (from 0 to 7 weeks' delay), and the strong association remained for 7-8 weeks. The effect of temperature on HFMD transmission could be divided into three phases (Fig. 4).

Phase 1: coupling. These was no significant similarity between the tracks of the temperature indicators and the incidence of HFMD from 0 to 7 weeks' delay. The similarity between the tracks increased over time, while this pattern was the opposite for other climate indicators.

Phase 2: coupled. With a time lag from 7 to 15 weeks, the similarity indices between the tracks of the mass centres of HFMD and temperature reached approximately 0.8 , which indicates that they were highly coupled at the time lags.

Phase 3: decoupling. When the time lag was longer than 15 weeks, the similarity indices between the tracks of the mass centres of HFMD and temperature decreased, which suggests a decreased effect of temperature on HFMD transmission.

The association of HFMD with other climate indicators (air pressure, wind speed, sunshine, humidity and precipitation) could be divided into two periods (Fig. 4).

Period 1 with a weak effect. At the start of a 9week outbreak period, the similarity indices were between 0.3 and 0.7 , suggesting that there was no significant association between the incidence of HFMD and these climate indictors. Wind and precipitation had a higher similarity with the incidence of HFMD than other climate indicators, which implies that these two climatic variables had more effect on HFMD transmission.

Period 2: recession. Nine weeks later, the mass centre tracks of climate indicators and the incidence of HFMD became less similar, indicating that the effect of the climate indictors on HFMD decreased during this period. Fig. 5 shows that a temporal trend $\left(a_{4}\right)$ of HFMD clustering occurred from May to August 2008 and then dispersed by September. However, clustering reappeared in the period December 2008 to March 2009 (latest available data). The spatial pattern was the same, but it had an approximate 7-week time lag with respect to the temperature indictors. The temperature variation was well matched with the temporal trend of HFMD at the time lag of seven weeks.

\section{Discussion}

According to Fraser et al. (2009), the evolution of the spatial macro patterns of HFMD incidence is due to external manifestation of virus micro movements. Both human outdoor activities and enterovirus mobility increase with higher temperatures, thereby augmenting the possibility for HFMD contagion. The dynamics of spatial clustering of air pressure and sunshine hours are similar to those of temperature because these two climate indictors have a strong association with temperature. The 7-week delay in HFMD 
a

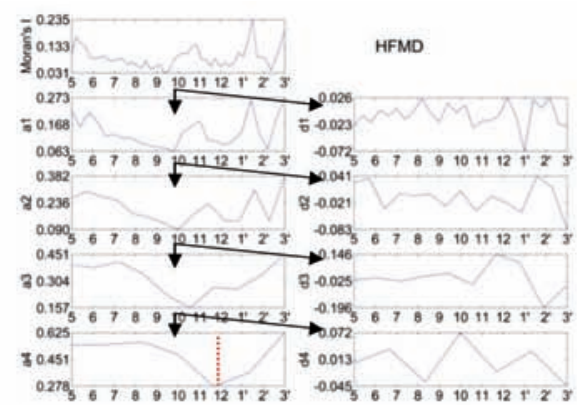

b

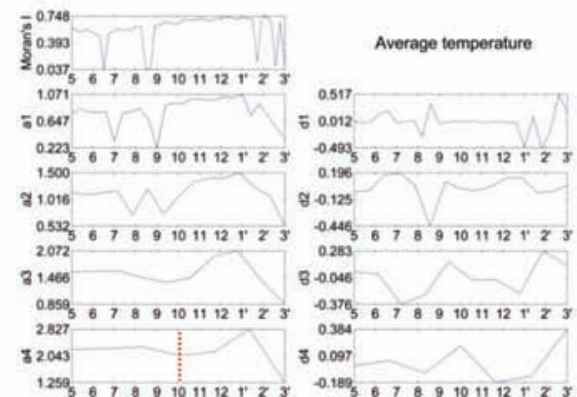

c

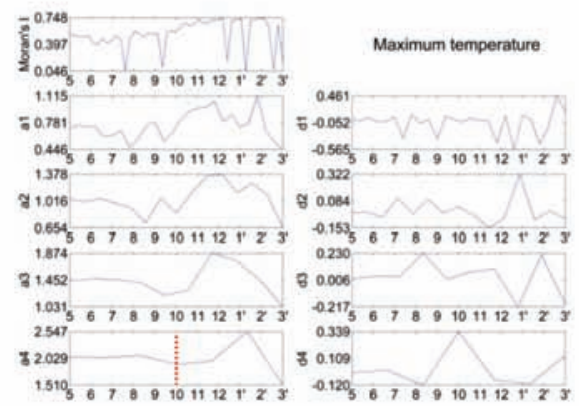

d

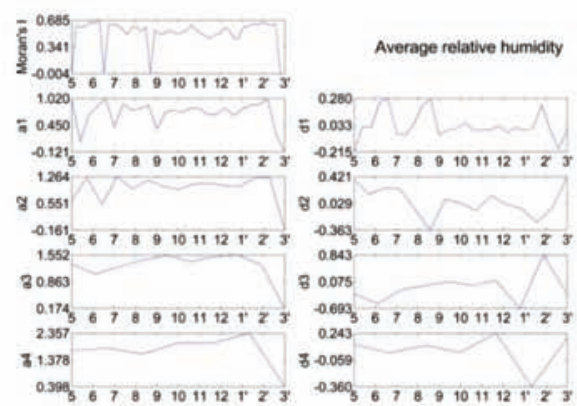

e

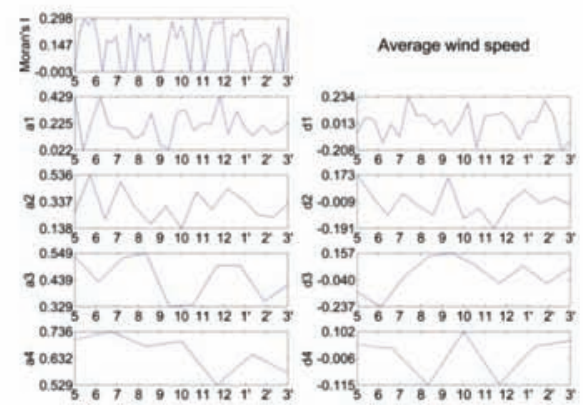

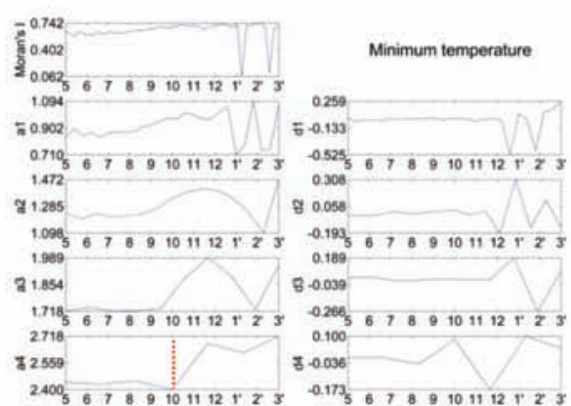
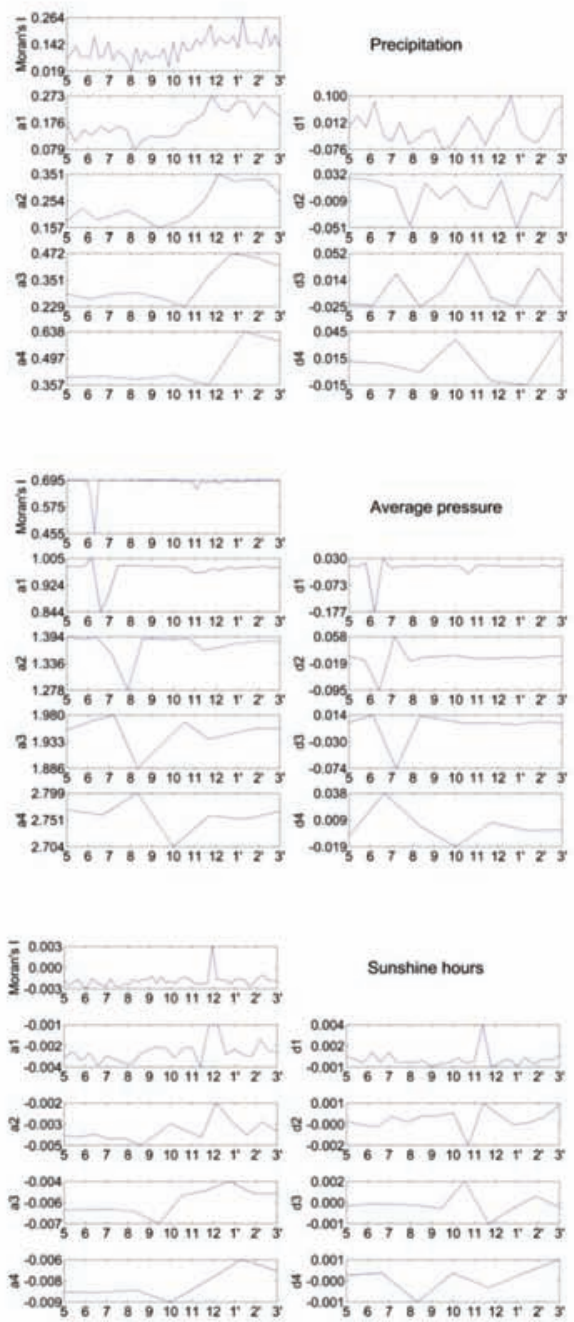

Fig. 5. Wavelet decomposition of Moran's I values of HFMD and climate indicators (the red lines mark the lowest spatial clustering of HFMD in late November and of the major climate indicators in October, respectively). 
Table 2. States and intervals from the point of climate variation to the disease response.

\begin{tabular}{l}
\hline State \\
A: Temperature variation \\
B: HFMD enterovirus responses to temperature variation
\end{tabular}

a(Goh et al., 1982; Garner and Lack, 1995); '(Alsop et al., 1960; Chang et al., 2002); '(Alsop et al., 1960); '(Wang et al., 2011).

temperature response is composed of five temporal intervals between six sequential states (Table 2). At any particular temporal cross-section, some area is dominated by either one of the states.

We examined whether there are interactions between monthly temperature, precipitation, and total solar radiation, and their effect on HFMD spatial transmission. Table 3 shows that the $P D$ values of an interaction between any two of the climate indicators are larger than any single value, which suggests that the climate indicators interact and enhance their effect on the spatial transmission of HFMD. The interaction between temperature and other climate indicators had a significantly greater effect than any other interaction on spatial transmission of HFMD. The PD values of climate in spring and autumn were higher than those in summer and winter. This finding indicates that spatially stratified non-homogeneity of climate in spring and autumn strongly controls the spatial dynamics of HFMD. Also, the disease transmission is less sensitive to climate change during the summer and winter than in the spring and autumn.

The statistical approach is good at summarizing the features of spatio-temporal phenomena and also for interpolating the processes based on spatio-temporal autocorrelation. However, it is limited with respect to the understanding of the physical mechanism at work and also limited with regard to interpretation of the biological principle of the 7-week delay of HFMD transmission with temperature variation. Mathematical modelling of a disease process, including the mechanism of transmission, can be employed to extract epidemiological parameters (Wang et al., 2006, 2011a), and it has been successfully applied to simulate scenarios under different controlling strategies for many diseases, such as mouth-foot disease (Keeling et al., 2003), influenza (Ferguson et al., 2005) and smallpox (Riley and Ferguson, 2006). The mathematical modelling approach deserves to be applied to HFMD. The chal-

Table 3. Climate indicators associated with HFMD transmission.

\begin{tabular}{lcccccc}
\hline & \multicolumn{7}{c}{ Climate factors } \\
\cline { 2 - 7 } Year/month & $\mathrm{A}$ & $\mathrm{B}$ & $\mathrm{C}$ & $\mathrm{A} \cap \mathrm{B}$ & $\mathrm{A} \cap \mathrm{C}$ & $\mathrm{B} \cap \mathrm{C}$ \\
\hline $2008-05$ & 0.043 & 0.119 & 0.041 & 0.178 & 0.141 & 0.201 \\
$2008-06$ & 0.040 & 0.049 & 0.049 & 0.144 & 0.116 & 0.169 \\
$2008-07$ & 0.017 & 0.019 & 0.012 & 0.103 & 0.077 & 0.088 \\
$2008-08$ & 0.015 & 0.031 & 0.090 & 0.219 & 0.316 & 0.352 \\
$2008-09$ & 0.026 & 0.112 & 0.054 & 0.460 & 0.307 & 0.347 \\
$2008-10$ & 0.010 & 0.089 & 0.042 & 0.194 & 0.157 & 0.197 \\
$2008-11$ & 0.029 & 0.111 & 0.017 & 0.315 & 0.131 & 0.320 \\
$2008-12$ & 0.015 & 0.025 & 0.017 & 0.138 & 0.131 & 0.116 \\
$2009-01$ & 0.045 & 0.069 & 0.022 & 0.263 & 0.184 & 0.220 \\
$2009-02$ & 0.028 & 0.104 & 0.040 & 0.226 & 0.141 & 0.262 \\
$2009-03$ & 0.060 & 0.087 & 0.102 & 0.311 & 0.257 & 0.248 \\
\hline
\end{tabular}

$\mathrm{A}=$ monthly average precipitation; $\mathrm{B}=$ monthly average temperature $\mathrm{C}=$ monthly total solar radiation; $\cap=$ interaction. 
lenges include multi-source endemics of HFMD (Wang et al., 2011a) and parameters associated with the variation of external determinants, e.g. human movements, weather and change in land use $(\mathrm{Hu}$ et al., 2012).

\section{Conclusions}

Understanding the connection between disease and environmental factors should be helpful for the treatment of a disease. The finding of a delay of 7 weeks between HFMD spatial distribution and any temperature change, bridges the gap between disease prediction and weather prediction. Moreover, it permits forecasting the HFMD transmission based on spatiotemporal temperature variation, which may be estimated by atmospheric science and meteorological forecasting. Intervention strategies would be much more efficient if they could be customised to spatial clustering and disease dispersal during different seasons. Consequently, intervention and prevention measures should predominantly focus on vulnerable populations, such as kindergartens and junior schools located in HFMD risk areas during periods of high risk.

\section{Acknowledgements}

This study was supported by MOST (2012CB955503; 2012ZX10004-201; 2011AA120305; 201202066), CAS (XDA05090102) and NSFC (41023010; 41271404).

\section{References}

Alsop J, Flewett TH, Foster JR, 1960. Hand-foot-and-mouth disease in Birmingham in 1959. Brit Med J 2, 1708-1711.

Alt H, Godau M, 1995. Computing the Fréchet distance between two polygonal curves. Int J Comput Geom Ap 5, 75 91.

Chang LY, King CC, Hsu KH, Ning HC, Tsao KC, Li CC, 2002. Risk factors of enterovirus 71 infection and associated hand, foot, and mouth disease/herpangina in children during an epidemic in Taiwan. Pediatrics 109, e88.

Christakos G, 2005. Interdisciplinary public health reasoning and epidemic modelling: the case of black death. New York: Springer Verlag.

Christakos G, 2010. Integrative problem-solving in a time of decadence. New York: Springer Verlag.

Christakos G, Hristopulos DT, 1998. Spatiotemporal environmental health modelling: a tractatus stochasticus. Boston: Kluwer Academic Publishers.

Eiter T, Mannila H, 1994. Computing discrete Fréchet distance, Technical Report CD-TR 94/64, Information Systems
Department, Technical University of Vienna, Vienna, Austria. Ferguson NM, Cummings DAT, Cauchemez S, Fraser C, Riley S, Meeyai A, 2005. Strategies for containing an emerging influenza pandemic in Southeast Asia. Nature 437, 209-214.

Fraser C, Donnelly CA, Cauchemez S, Hanage WP, Van Kerkhove MD, Hollingsworth TD, 2009. Influenza: making privileged data public response. Science 325, 1072-1073.

Garner M, Lack M, 1995. An evaluation of alternate control strategies for foot-and-mouth disease in Australia: a regional approach. Prev Vet Med 23, 9-32.

Goh KT, Doraisingham S, Tan JL, Lim GN, Chew SE, 1982. An outbreak of hand, foot, and mouth-disease in Singapore. Bull World Health Organ 60, 965-969.

Haining RP, 2003. Spatial data analysis: theory and practice. Cambridge: Cambridge University Press.

Hii YL, Rocklov J, Ng N, 2011. Short term effects of weather on hand, foot and mouth disease. PLoS One 6, e16796.

Hu MG, Li ZJ, Wang JF, Jia L, Liao Y, 2012. Determinants of the incidence of Hand, Foot and Mouth Disease in China using geographically weighted regression models. PLoS One 7, e38978.

Keeling MJ, Woolhouse MEJ, May RM, Davies G, Grenfell BT, 2003. Modelling vaccination strategies against foot-andmouth disease. Nature 421, 136-142.

Kolovos A, Skupin A, Jerrett M, Christakos G, 2010. Multi-perspective analysis and spatiotemporal mapping of air pollution monitoring data. Environ Sci Technol 44, 6738-6744.

Kulldorff M, 1997. A spatial scan statistic. Commun Stat Theory 26, 1481-1496.

Moran PAP, 1950. Notes on continuous stochastic phenomena. Biometrika 37,17-23.

Riley S, 2008. A prospective study of spatial clusters gives valuable insights into dengue transmission. PLoS Med 5, 15401541.

Riley S, Ferguson NM, 2006. Smallpox transmission and control: spatial dynamics in Great Britain. Proc Natl Acad Sci USA 103, 12637-12642.

Wang JF, 2009. Qualitative and quantitative analysis of map. Journal of Geo-Information Science 11, 169-175 (in Chinese). Wang JF, Guo YS, Christakos G, Yang WZ, Liao YL, Li ZJ, 2011a. Hand, foot and mouth disease: spatiotemporal transmission and climate. Int J Health Geogr 10, 25.

Wang JF, Haining R, Cao ZD, 2010a. Sample surveying to estimate the mean of a heterogeneous surface: reducing the error variance through zoning. Int J Geogr Inf Sci 24, 523-543.

Wang JF, Hu Y, 2012. Environmental health risk detection with GeogDetector. Environ Modell Softw 33, 114-115.

Wang JF, Li XH, Christakos G, Liao YL, Zhang T, Gu X, 2010b. Geographical detectors-based health risk assessment and its application in the neural tube defects study of the Heshun region, China. Int J Geogr Inf Sci 24, 107-127.

Wang JF, McMichael AJ, Meng B, Becker NG, Han WG, Glass 
K, 2006. Spatial dynamics of an epidemic of severe acute respiratory syndrome in an urban area. Bull World Health Organ 84, 965-968.

Wang Y, Feng ZJ, Yang Y, Self S, Gao YJ, Longini IM, 2011b. Hand, foot, and mouth disease in China: patterns of spread and transmissibility. Epidemiology 22, 781-792.

Yu HL, Yang SJ, Yen HJ, Christakos G, 2011. A spatio-tempo- ral climate-based model of early dengue fever warning in southern Taiwan. Stoch Env Res Risk A 25, 485-494.

Zhang J, Sun JL, Chang ZR, Zhang WD, Wang ZJ, Feng ZJ, 2011. Characterization of hand, foot, and mouth disease in China between 2008 and 2009. Biomed Environ Sci 24, 214221.

Zhou XN, 2009. Spatial epidemiology. Beijing: Science Press. 